\title{
Evaluation of Window Size in Classification of Epileptic Short-Term EEG Signals Using a Brain Computer Interface Software
}

\author{
Katerina D. Tzimourta \\ Medical Physics Laboratory, \\ University of Ioannina \\ Ioannina, Greece \\ ktzimourta@cc.uoi.gr
}

\author{
Nikolaos Giannakeas \\ Computer Engineering Dpt, \\ Technological Educational \\ Institute of Epirus, \\ Kostakioi, Arta, Greece \\ giannakeas@teiep.gr
}

\author{
Loukas G. Astrakas \\ Medical Physics Laboratory, \\ University of Ioannina \\ Ioannina, Greece \\ astrakas@uoi.gr
}

\author{
Anna Maria Gianni \\ Computer Engineering Dpt, \\ Technological Educational \\ Institute of Epirus, \\ Kostakioi, Arta, Greece \\ anna.maria.gianni@gmail.com
}

\author{
Dimitrios G. Tsalikakis \\ Informatics and \\ Telecommunications Eng. \\ Dpt, University of Western \\ Macedonia, Kozani, Greece \\ dtsalikakis@uowm.gr
}

\author{
Alexandros T. Tzallas \\ Computer Engineering Dpt, \\ Technological Educational \\ Institute of Epirus, \\ Kostakioi, Arta, Greece \\ tzallas@teiep.gr
}

\author{
Markos G. Tsipouras \\ Informatics and \\ Telecommunications Eng. \\ Dpt, University of Western \\ Macedonia, Kozani, Greece \\ mtsipouras@uowm.gr
}

\begin{abstract}
The complexity of epilepsy created a fertile ground for further research in automated methods, attempting to help the epileptologists' task. Over the past years, great breakthroughs have emerged in computer-aided analysis. Furthermore, the advent of Brain Computer Interface (BCI) systems has facilitated significantly the automated seizure analysis. In this study, an evaluation of the window size in automated seizure detection is proposed. The EEG signals from the University of Bonn was employed and segmented into 24 epochs of different window lengths with $50 \%$ overlap each time. Statistical and spectral features were extracted in the OpenViBE scenario and were used to train four different classifiers. Results in terms of accuracy were above $80 \%$ for the Decision Tree classifier. Also, results indicated that different window sizes provide small variations in classification accuracy.
\end{abstract}

Keywords-epilepsy; EEG; seizure detection; window size; brain computer interface

\section{INTRODUCTION}

Epilepsy is a devastating brain disorder followed by seizures, which are repetitive episodes of temporary interruption or disturbance of communication between neurons. Many people experience seizures without a clear cause and almost one-third of the epileptic patients suffer from refractory seizures [1]. The latest facts render epilepsy a life-threatening disorder and a major factor responsible for mortality in developed and developing countries [2]. Depending on the brain areas that participate during epileptic activity, seizures are divided into two fundamental types: partial (affects only a single brain area) and generalized (affects more than one region). These two main types are subdivided forming a bigger list of several seizure types [3]. The Electroencephalogram (EEG) is used to monitor and diagnose epilepsy. The brain activity is monitored through the EEG which is usually performed in a well-equipped hospital. The electrodes are either attached to the surface of the skull (scalp EEG - sEEG) or placed invasively inside the brain (intracranial EEG iEEG). Furthermore, the EEG recording is usually performed after a seizure episode and before the next seizure occurrence (interictal period). Rarely an EEG recording captures the seizure onset (ictal period) and it usually happens in a 24-hour monitoring. The complexity of EEG recordings and the huge amount of data, led to the development of methods for detecting different patterns of brain activity [4] and automated seizure analysis [5]. Generally, these methods follow a pattern recognition approach, which contains feature extraction and classification. The signal is usually decomposed in epochs of specific duration in an attempt to better capture the transient occurrences of the EEG. Several time-frequency analysis methods have been proposed such as Discrete Wavelet Transform [6-8], Wigner-Ville distribution [9], Empirical Mode Decomposition [10-11] etc. Significant features are extracted from the decomposed signal and are then used to train a classifier. Usually, the raw signal is initially analyzed in epochs of small duration and the window size is of primary importance in automated seizure detection.

Recent breakthroughs in computer-aided analysis initiated the development of Brain Computer Interface (BCI) systems, which are more user-friendly and provide direct communication with the user's brain in real-time without any possible movement [12]. In this work, a method for seizure 
detection and the investigation of window size in signal analysis is presented. The EEG recordings from a public available database was used and decomposed in epochs of different lengths, ranging from 1 to 24 seconds with $50 \%$ overlap. Significant statistical and spectral features were calculated and employed to train four classifiers. In total, 24 cases were created in the open-source software OpenViBE that trained and tested the four classifiers.

\section{MATERIALS AND METHOD}

In this work, the OpenViBE BCI software was used for signal processing and the WEKA environment for training and testing various classifiers. The OpenViBE [13] is an easy, open-source software designed to be used for direct communication with the brain, by a broad range of users with minimum of programming skills. To investigate the role of the window size in EEG signal analysis, the database of the University of Bonn was used. The EEG signals were decomposed in various epochs of different sizes and several features were extracted in the OpenViBE scenario. These features were used to train four remarkable classifiers. In Figure 1 a flowchart of the proposed method is presented. Also, an example of the OpenViBE scenario that contains the training parameters which used to train the classifiers is depicted in Figure 2.

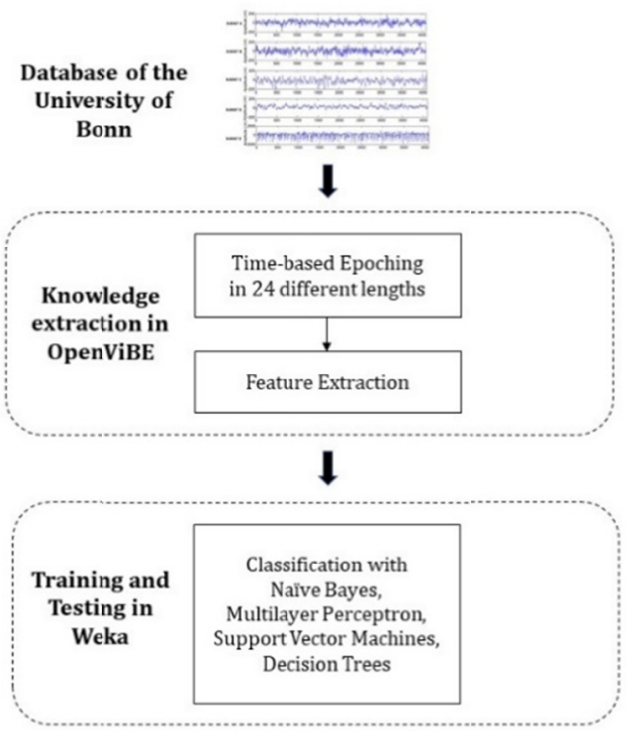

Fig. 1. A flowchart of the proposed three-step methodology

\section{A. The Database}

The database University of Bonn [14] was employed in order to investigate the window length. The database consists of EEG segments that were selected and cut out of continuous EEG recordings, which were acquired from 5 healthy volunteers and 5 epileptic patients. The EEG segments are grouped in five subsets, denoted as A, B, C, D and E each containing of 100 single-channel EEG segments of 23.6-second duration marked as $\mathrm{Z}, \mathrm{O}, \mathrm{N}, \mathrm{F}$ and $\mathrm{S}$, respectively. The sampling frequency of the data is $173.61 \mathrm{~Hz}$ and any artifacts were visually removed. More details about the database are presented in Table I. In this study, all the 500 segments were employed to create the problem of five classes, wherein each set is a class (Z-O-N-F-S). This particular problem is more challenging and many studies [15-17] have attempted to obtain as good classification results as the ones obtained from a binary problem.

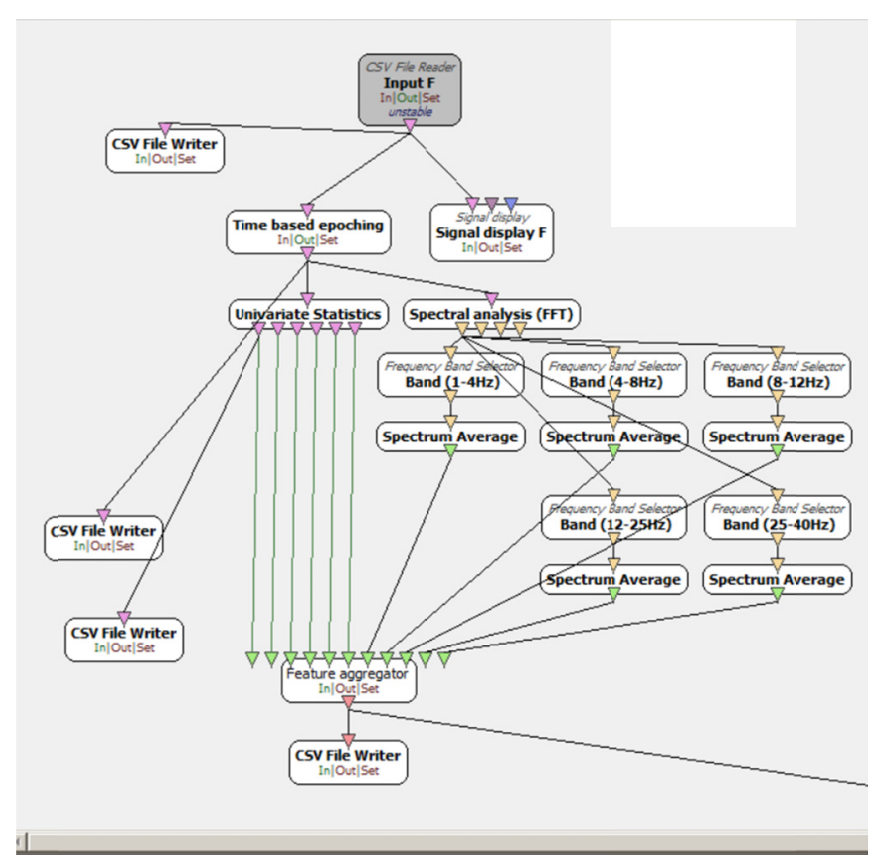

Fig. 2. Part of the OpenViBE scenario for the "F" EEG segments.

TABLE I. SUMMARY OF THE BONN DATABASE

\begin{tabular}{|c|c|c|c|}
\hline $\begin{array}{c}\text { Subset } \\
\text { label }\end{array}$ & $\begin{array}{l}\text { Type of } \\
\text { EEG } \\
\text { recording }\end{array}$ & Subjects & Subject's state \\
\hline Z & sEEG & healthy & $\begin{array}{c}\text { relaxed in an awake state with eyes } \\
\text { opened }\end{array}$ \\
\hline $\mathrm{O}$ & sEEG & healthy & $\begin{array}{c}\text { relaxed in an awake state with eyes } \\
\text { closed }\end{array}$ \\
\hline $\mathrm{N}$ & iEEG & epileptic & $\begin{array}{c}\text { Seizure-free from the opposite } \\
\text { hemisphere of the epileptogenic } \\
\text { zone }\end{array}$ \\
\hline $\mathrm{F}$ & iEEG & epileptic & $\begin{array}{l}\text { Seizure-free from the epileptogenic } \\
\text { zone }\end{array}$ \\
\hline S & iEEG & epileptic & $\begin{array}{c}\text { Seizure activity from the } \\
\text { epileptogenic zone }\end{array}$ \\
\hline
\end{tabular}

\section{B. Time Epoching}

In the OpenViBE Designer the EEG signals were decomposed in epochs of consecutive seconds, starting from 1 second with 0.5 seconds overlap. The plugin "Time based epoching" was used to configure the duration of the EEG epochs from 1 to 24 seconds with 50\% seconds epoch overlap each time. In total, 24 XML files were created with EEG segments ranging from 1 second up until 24 seconds with 0.5 13 seconds overlap. 


\section{Feature Extraction}

A set of 8 features was extracted in each epoch, forming the feature vector that used in the classification. The plugins "Univariate Statistics" and "Spectral Analysis" were employed in the OpenViBE scenario to compute four time-based features namely:

- mean value,

- variance,

- range (maximum value - minimum value),

- median value,

and four spectral features based on Fast Fourier Transform being:

- the spectrum amplitude (the power of the signal) in alpha band $(8-12 \mathrm{~Hz})$

- the spectrum amplitude in beta band $(12-25 \mathrm{~Hz})$,

- the spectrum amplitude in theta band (4-8Hz),

- the spectrum amplitude in delta band (1-4Hz),

The resulting feature set was used to train four well-known classifiers, being Naïve Bayes, Multilayer Perceptron, Support Vector Machines and Decision Tree.

\section{Classification}

To evaluate the proposed method and the window size in classification results, four of the most sophisticated supervised classifiers were used.

\section{1) Nä̈ve Bayes (NB)}

Naïve Bayes (NB) is a simple classifier that combines a probability model with a decision rule. The classifier operates on the simplest assumption that the features are conditionally independent and is based on Bayes decision theory, wherein the posterior probability of each class is calculated by the likelihood and the prior probability [15]. The ultimate goal of the classifier is to minimize the probability of classification error and maximize the posterior probability. A small number of training data is needed for the classifier to estimate the necessary classification parameters and in conjunction with the less time complexity, this simple classifier has preferred in complex classification problems.

\section{2) MultiLayer Perceptron (MLP)}

The Multilayer Perceptron (MLP) classifier is a neural network with at least three layers of nodes. MLP utilizes the backpropagation techniques for training and maps non-linear input data into a space, where it becomes linearly separable. In order to train a MLP classifier and perform correct pattern classification, the connection weights after each processing of data are adjusted, based on the comparison between the error in the output and the expected result [15].

\section{3) Support Vector Machines (SVM)}

Support Vector Machines is a machine learning technique for linear and non-linear classification problems. The nonlinear input data is projected into a high-dimension feature space in order to be linearly separated. This projection is performed by the kernel function, which can be either a linear or a polynomial function, the radial basis function or the sigmoid kernels. The gap that separates the data is called hyperplane and the major goal of the algorithm is to find the optimal separating hyperplane that maximizes the distance between the data and minimizes the classification error [15]. In our experiments, the radial basis function was used.

\section{4) Decision Tree (DT)}

A Decision Tree (DT) classifier is a straightforward classifier based on a series of decision rules. The root node of the tree is displayed at the top and is successively connected with other nodes through links or branches, until no further links to other nodes exist (leaf nodes). According to the DT classifier, only one link can be followed each time and the subsequent node becomes the root node of the next sub-tree. The procedure is repeated until a leaf node is reached, leading to no further decision and the category label is read [15].

\section{RESULTS AND DISCUSSION}

The four classifiers were trained and tested according to the 10-fold cross-validation technique. To evaluate the classification results of the experiments and thus, the window size the accuracy was calculated from the correctly classified instances:

$$
\text { Accuracy }=\frac{\text { correctly classified instances }}{\text { total number of instances }}
$$

The obtained statistical results of the 24 experiments for each classifier are presented in Table II.

TABLE II. RESULTS

\begin{tabular}{|c|c|c|c|c|c|}
\hline \multirow{2}{*}{$\begin{array}{c}\text { Epoch } \\
\text { (sec) }\end{array}$} & \multirow{2}{*}{$\begin{array}{l}\text { Over- } \\
\text { lapping } \\
\text { (sec) }\end{array}$} & \multicolumn{4}{|c|}{ Correctly Classified instances (\%) } \\
\hline & & NB & MLP & SVM & DT \\
\hline 1 & 0.5 & $59.11 \%$ & $72.41 \%$ & $67.51 \%$ & $85.95 \%$ \\
\hline 2 & 1.0 & $63.67 \%$ & $75.56 \%$ & $70.35 \%$ & $79.06 \%$ \\
\hline 3 & 1.5 & $66.37 \%$ & $78.02 \%$ & $70.46 \%$ & $82.05 \%$ \\
\hline 4 & 2.0 & $67.47 \%$ & $78.73 \%$ & $70.11 \%$ & $83.50 \%$ \\
\hline 5 & 2.5 & $68.97 \%$ & $79.47 \%$ & $68.87 \%$ & $83.60 \%$ \\
\hline 6 & 3.0 & $70.32 \%$ & $81.72 \%$ & $70.00 \%$ & $84.87 \%$ \\
\hline 7 & 3.5 & $70.23 \%$ & $81.10 \%$ & $69.21 \%$ & $85.45 \%$ \\
\hline 8 & 4.0 & $70.03 \%$ & $80.17 \%$ & $68.55 \%$ & $85.17 \%$ \\
\hline 9 & 4.5 & $71.90 \%$ & $80.91 \%$ & $68.45 \%$ & $85.19 \%$ \\
\hline 10 & 5.0 & $72.11 \%$ & $77.75 \%$ & $63.63 \%$ & $83.83 \%$ \\
\hline 11 & 5.5 & $72.08 \%$ & $83.15 \%$ & $68.10 \%$ & $85.48 \%$ \\
\hline 12 & 6.0 & $73.33 \%$ & $81.95 \%$ & $68.46 \%$ & $85.39 \%$ \\
\hline 13 & 6.5 & $73.52 \%$ & $81.03 \%$ & $67.44 \%$ & $83.72 \%$ \\
\hline 14 & 7.0 & $74.01 \%$ & $81.23 \%$ & $67.83 \%$ & $84.25 \%$ \\
\hline 15 & 7.5 & $75.29 \%$ & $81.98 \%$ & $68.34 \%$ & $82.71 \%$ \\
\hline 16 & 8.0 & $75.61 \%$ & $83.35 \%$ & $66.57 \%$ & $84.51 \%$ \\
\hline 17 & 8.5 & $74.58 \%$ & $83.53 \%$ & $68.19 \%$ & $83.75 \%$ \\
\hline 18 & 9.0 & $75.85 \%$ & $82.39 \%$ & $67.72 \%$ & $82.31 \%$ \\
\hline 19 & 9.5 & $75.37 \%$ & $82.18 \%$ & $68.31 \%$ & $82.60 \%$ \\
\hline 20 & 10.0 & $75.57 \%$ & $83.00 \%$ & $67.87 \%$ & $81.23 \%$ \\
\hline 21 & 10.5 & $81.23 \%$ & $82.79 \%$ & $67.62 \%$ & $84.46 \%$ \\
\hline 22 & 11.0 & $76.48 \%$ & $82.04 \%$ & $68.87 \%$ & $82.82 \%$ \\
\hline 23 & 11.5 & $77.34 \%$ & $82.24 \%$ & $68.16 \%$ & $83.77 \%$ \\
\hline 24 & 12.0 & $77.55 \%$ & $81.70 \%$ & $68.82 \%$ & $85.63 \%$ \\
\hline
\end{tabular}


The best classifier for epochs of 1 second with 0.5 seconds overlap, is DT with $85.95 \%$ followed by MLP with $72.41 \%$, SVM with $67.51 \%$ and NB with $59.11 \%$. For epochs lasting 2 seconds with 1 second overlap, the best classifier is DT with 79.06\%, followed by MLP (75.56\%) and SVM (70.35\%) and the worst classifier is NB with $63.67 \%$ accuracy. For windows of 3 seconds with 1.5 seconds overlap and 4 seconds with 2 seconds overlap, DT remains the best classifier $(82.05 \%$ and $83.50 \%$ respectively) and the lowest accuracy $(66.37 \%$ and $67.47 \%$ respectively) is obtained with NB as well. For the next 12 window sizes the DT outperforms (ranging from $82.71 \%$ to $85.48 \%$ ) and the lowest values (ranging from $63.63 \%$ to $70 \%$ ) are obtained with SVM. At the same time, MLP indicates accuracy ranging from $77.75 \%$ to $83.35 \%$, whereas NB shows lower accuracy, ranging from $68.97 \%$ to $75.61 \%$, and in some cases perform almost the same with SVM (for windows of $5 \mathrm{sec}$ and 6 sec duration). For the next 4 window sizes the DT and the MLP presents almost the same accuracy and none of the two classifiers is considerably better than the other. Accuracy for NB is about $75 \%$ for these four window sizes and SVM does not exceed $68 \%$. Finally, for the last four window sizes, DT is the best classifier (ranging from $81.23 \%$ to $85.63 \%$ ) followed by MLP (ranging from $82.04 \%$ to $83 \%$ ), NB (ranging from $75.57 \%$ to $81.23 \%$ ) and SVM (ranging from $67.62 \%$ to $68.87 \%$ ). The smooth and small changes of the obtained results for each classifier and the comparison between their performances are depicted in Figure 3. From Figure 3 it can be observed that while the window size is increasing, the classification results for NB, MLP and DT are slightly higher, without great variations (in most cases not greater that 3\%). The lowest accuracy for NB and MLP is $59.11 \%$ and $72.41 \%$ and the highest $81.23 \%$ and $83.53 \%$, respectively; however, despite the big difference between the lowest and highest values, the intermediate values range from $1 \%$ to $3 \%$. The best classification accuracy is provided with DT with lowest value $79.06 \%$ ( 2 seconds window with 1 second overlap) and highest $85.63 \%$ (24 seconds with 12 seconds overlap) whereas SVM showed the worst classification accuracy (lowest value 63.63\% and highest $70.46 \%$ ). Also, the best accuracy for DT, MLP and NB (above $80 \%$ ) is obtained for windows of 21 seconds with 10.5 seconds overlap, whereas the SVM seemed to be the weakest classifier compared to the others.

The epoch duration has also been a focus point for several researchers. Recently [16], a method based on dynamic principal component analysis (DPCA) and energy was proposed. The authors evaluated four window sizes of the EEG segments, being 64, 128, 256 and 512 samples per window (approximately $0.37,0.74,1.47$ and 2.95 seconds respectively). Results showed minor increase when signals were segmented in 512 samples epochs per window. In [17], authors conducted 4 experiments to find the optimal window size between 4 options. In the presented method the Permutation Entropy was calculated from nonoverlapping epochs of $0.25,0.5,0.75$ and 1 second and was used to train a SVM classifier. Results in terms of F1 score showed small deviation (about 5\% for overall F1 score). An extension of the above mentioned approach was presented in [18] wherein the same group of authors investigated among other, whether the overlapping plays significant role in seizure detection or not and they proposed a method based on Weighted Permutation Entropy (WPE). In this approach, 200 signals of the Bonn database were employed and segmented in epochs of approximately 0.35 seconds duration with overlap (OV) (128 samples per window) and without overlap (NOV) (164 samples per window). The method was evaluated on SVM and Artificial Neural Network (ANN) classifier and results indicated that epochs with 50\% overlap provided slightly higher accuracy $(2.25 \%$ overall accuracy for SVM and ANN). In our experiments, 24 window sizes are evaluated and the classification accuracy slightly arises as the window size increases for all classifiers. The best classifier is DT with accuracy ranging from $79.06 \%$ to $85.95 \%$, followed by MLP ranging from $72.41 \%$ to $83.53 \%$, SVM from $67.51 \%$ to $70.46 \%$ and $\mathrm{NB}$ from $59.11 \%$ to $75.61 \%$. A comparison table is presented in Table III.

TABLE III. COMPARISON TABLE

\begin{tabular}{|c|c|c|c|c|c|c|}
\hline Reference & Window size & Signal analysis & Features & Classifier & $\begin{array}{l}\text { Classification } \\
\text { Problem }\end{array}$ & Performance Metrics \\
\hline$[16]$ & $\begin{array}{l}4 \quad \text { non-overlapping } \\
\text { window sizes ranging } \\
\text { from } \\
0.37-2.95 \mathrm{sec}\end{array}$ & $\begin{array}{l}\text { Dynamic Principal } \\
\text { Component Analysis }\end{array}$ & Energy & $1-\mathrm{NN}$ & $\begin{array}{l}\text { Z-S } \\
\text { ZONF-S }\end{array}$ & Accuracy: $99.9 \%-100 \%$ \\
\hline$[17]$ & $\begin{array}{l}4 \text { non-overlapping } \\
\text { window sizes ranging } \\
\text { from } \\
0.25-1 \mathrm{sec}\end{array}$ & Raw segmented data & $\begin{array}{l}\text { Weighted Permutation } \\
\text { Entropy }\end{array}$ & SVM & $\begin{array}{l}\mathrm{Z}-\mathrm{S} \\
\mathrm{O}-\mathrm{S} \\
\mathrm{N}-\mathrm{S} \\
\mathrm{F}-\mathrm{S}\end{array}$ & $\begin{array}{l}\text { Average F1 values for each } \\
\text { window size: } 0.866-0.917\end{array}$ \\
\hline \multirow{2}{*}[18]{} & $\begin{array}{l}0.35 \text { sec non- } \\
\text { overlapping window }\end{array}$ & \multirow{2}{*}{ Raw segmented data } & \multirow{2}{*}{$\begin{array}{l}\text { Weighted } \\
\text { Entropy }\end{array}$} & \multirow[t]{2}{*}{ SVM } & \multirow{2}{*}{ Z-O-N-F-S } & $\begin{array}{l}\text { NOV Accuracy: } 90.63 \text { - } \\
92.88 \%\end{array}$ \\
\hline & $\begin{array}{l}0.35 \text { sec with } 50 \% \\
\text { overlap window }\end{array}$ & & & & & $\begin{array}{l}\text { OV Accuracy: } 91.63 \text { - } \\
93.88 \%\end{array}$ \\
\hline \multirow{4}{*}{$\begin{array}{l}\text { This } \\
\text { work }\end{array}$} & \multirow{4}{*}{$\begin{array}{l}24 \quad \text { overlapping } \\
\text { windows with } 50 \% \\
\text { overlap ranging from 1- } \\
24 \mathrm{sec}\end{array}$} & \multirow{4}{*}{ Raw segmented data } & \multirow{4}{*}{$\begin{array}{l}\text { Mean, Range, Variance, } \\
\text { Median, Spectral } \\
\text { amplitude in alpha, beta, } \\
\text { delta, theta band }\end{array}$} & \multirow{4}{*}{$\begin{array}{l}\text { Naïve } \\
\text { Bayes, } \\
\text { MLP, } \\
\text { SVM, DT }\end{array}$} & \multirow{4}{*}{ Z-O-N-F-S } & $\begin{array}{l}\text { NB Accuracy: } 59.11 \% \quad- \\
81.23 \%\end{array}$ \\
\hline & & & & & & $\begin{array}{l}\text { MLP Accuracy: } 72.41 \% \text { - } \\
83.53 \%\end{array}$ \\
\hline & & & & & & $\begin{array}{l}\text { SVM Accuracy: } 63.63 \%- \\
70.46 \%\end{array}$ \\
\hline & & & & & & $\begin{array}{l}\text { DT Accuracy: } 79.06 \% \quad- \\
85.63 \%\end{array}$ \\
\hline
\end{tabular}




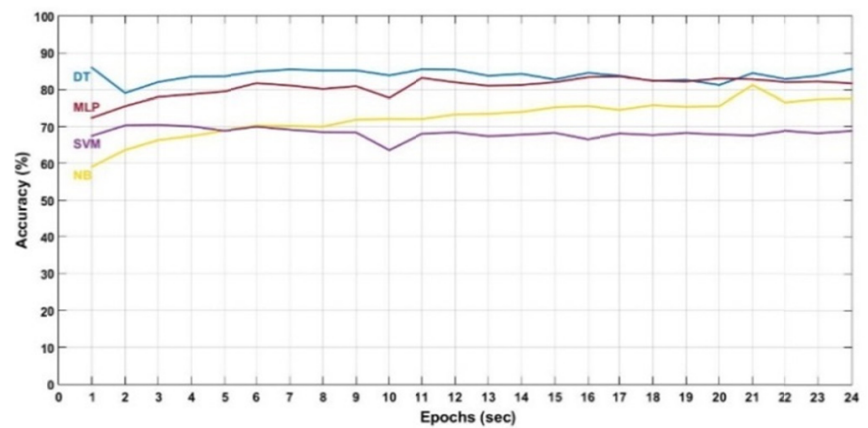

Fig. 3. Results in terms of accuracy for the 4 classifiers (blue: DT, red: MLP, yellow: NB, purple: SVM)

\section{CONCLUSIONS}

Epilepsy is constantly under the microscope and many studies have focused on seizure detection and prediction. In most of the studies, the authors follow a certain three-step methodology, which contain signal decomposition, feature extraction and classification. The window size in signal decomposition is very important on detecting the subtle changes of the EEG recording. However, results of our experiments indicated that small differences in epoch duration do not have a significant impact on the classification accuracy. Consequently, a smaller number of epochs decreases the computational time of the classification process which is a significant parameter in real-time applications [19] Furthermore, the best window size seemed to be the one of 21 seconds with 10.5 seconds overlap, which may be a promising window length for seizure prediction. Since the signal duration was 23.6 seconds, little do we know about the window size for above 24 seconds. In the future, the investigation of the window size can be performed in long-term EEG datasets, which are closer to the clinical EEG recordings.

\section{REFERENCES}

[1] World Health Organization, "Epilepsy", Fact sheet N999, 2012

[2] O. Devinsky, T. Spruill, D. Thurman, D. Friedman, "Recognizing and preventing epilepsy-related mortality: A call for action”, Neurology, Vol. 86, No. 8, pp. 779-786, 2016

[3] I. E. Scheffer, S. Berkovic, G. Capovilla, M. B. Connolly, J. French, L. Guilhoto, E. Hirsch, S. Jain, G. Mathern, S. Moshé, D. Nordli, EE. Perucca, T. Tomson, S. Wiebe, Y. Zhang, S. Zuberi, "ILAE classification of the epilepsies: Position paper of the ILAE Commission for Classification and Terminology", Epilepsia, Vol. 58, No. 4, pp. 512521,2017

[4] S. Segkouli, I. Paliokas, D. Tzovaras, M. Tsolaki, C. Karagiannidis, "Study of EEG Power Fluctuations Enhanced by Linguistic Stimulus for Cognitive Decline Screening", International Symposium on Pervasive Computing Paradigms for Mental Health, pp. 165-175, 2015

[5] A. T. Tzallas, M. G. Tsipouras, D. G. Tsalikakis, E. C. Karvounis, L. Astrakas, S. Konitsiotis, M. Tzaphlidou. "Automated epileptic seizure detection methods: a review study", in :Epilepsy, Intechopen, 2012.

[6] I. Guler, E. D. Ubeyli, "Multiclass support vector machines for EEGsignals classification", IEEE Transactions on Information Technology in Biomedicine, Vol. 11, No. 2, pp. 117-126, 2007

[7] Y. Kumar, M. L. Dewal, R. S. Anand, "Epileptic seizure detection using DWT based fuzzy approximate entropy and support vector machine", Neurocomputing, Vol. 133, pp. 271-279, 2014
[8] K. D. Tzimourta, L. G. Astrakas, M. G. Tsipouras, N. Giannakeas, A. T. Tzallas, S. Konitsiotis, "Wavelet based classification of epileptic seizures in EEG signals", 2017 IEEE 30th International Symposium on Computer-Based Medical Systems (CBMS), pp. 35-39, 2017

[9] A. T. Tzallas, M. G. Tsipouras, D. I. Fotiadis, "Automatic seizure detection based on time-frequency analysis and artificial neural networks", Computational Intelligence and Neuroscience, Vol. 18, pp. 113,2007

[10] S. S. Alam, M. I. H. Bhuiyan, "Detection of seizure and epilepsy using higher order statistics in the EMD domain", IEEE Journal of Biomedical and Health Informatics, Vol. 17, No. 2, pp. 312-318, 2013

[11] A. Bhardwaj, A. Tiwari, R. Krishna, V. Varma, "A novel genetic programming approach for epileptic seizure detection", Computer Methods and Programs in Biomedicine, Vol. 124, pp. 2-18, 2016

[12] L. Huang, G. van Luijtelaar, "Brain computer interface for epilepsy treatment", in: Brain-Computer Interface Systems-Recent Progress and Future Prospects, InTech, 2013

[13] Y. Renard, F. Lotte, G. Gibert, M. Congedo, E. Maby, V. Delannoy, O. Bertrand, A. Lécuyer, "OpenViBE: An Open-Source Software Platform to Design, Test and Use Brain-Computer Interfaces in Real and Virtual Environments1", Presence: Teleoperators and Virtual Environments, Vol. 19, 2010

[14] R. G. Andrzejak, K. Lehnertz, F. Mormann, C. Rieke, P. David, C. E Elger. "Indications of nonlinear deterministic and finite-dimensional structures in time series of brain electrical activity: Dependence on recording region and brain state", Physical Review E, Vol. 64, No. 6, 2001

[15] A.T. Tzallas, M. G. Tsipouras, D. I. Fotiadis, "Epileptic seizure detection in EEGs using time-frequency analysis", IEEE Transactions on Information Technology in Biomedicine, Vol. 13, No. 5, pp. 703-710, 2009

[16] C. Kamath. "Automatic seizure detection based on Teager Energy Cepstrum and pattern recognition neural networks", QScience Connect, Vol. 1, 2014

[17] A. Bhardwaj, A. Tiwari, R. Krishna, V. Varma, "A novel genetic programming approach for epileptic seizure detection", Computer methods and programs in biomedicine, Vol. 124, pp. 2-18, 2016

[18] R. O. Duda, P. E. Hart, D. G. Stork, Pattern Classification, John Wiley \& Sons, 2012

[19] S. Xie, S. Krishnan, "Dynamic principal component analysis with nonoverlapping moving window and its applications to epileptic EEG classification", The Scientific World Journal, Volume 2014, Article ID 419308, 2014

[20] N. Seddik, S. Youssef, M. Kholief, "Automatic seizure detection in long-term scalp EEG using Weighted Permutation Entropy and Support Vector Machine", Biomedical Engineering Conference (CIBEC), pp. 170-173, 2014

[21] N. S. Tawfik, S. M. Youssef, M. Kholief, "A hybrid automated detection of epileptic seizures in EEG records", Computers \& Electrical Engineering, Vol. 53, pp. 177-190, 2014

[22] V. Nagaraj, S. Lee, E. Krook-Magnuson, I. Soltesz, P. Benquet, P. Irazoqui, T. Netoff, "The Future of Seizure Pre-diction and Intervention: Closing the loop", Journal of Clinical Neurophysiology: Official Publication of the American Electroencephalographic Society, Vol. 32, No. 3, pp. 194,2015 